\title{
Was vom Tattoo übrig bleibt
}

\section{Entfernung mittels Laser hinterlässt gefährliche Spaltprodukte.}

\begin{abstract}
Wer sich tätowieren lässt, nimmt Risiken in Kauf, die zum Teil noch gar nicht näher erforscht sind. Wer sich von seinem Tattoo wieder trennen möchte - auch. Aktuelle Erkenntnisse aus der Lasertherapie, Tattoo-Entfernung mit dem Pikolaser, aber auch Komplikationen mit Tätowierungen, die sogar Jahre nach der Prozedur noch auftreten können, waren Themen bei der 9. Strategie-Sitzung der Deutschen Gesellschaft für Dermatochirurgie e.V. (DGDC) im Juni 2018 in Dresden.
\end{abstract}

\section{Tattoos und ihre Probleme}

Mögliche Langzeitfolgen können knotige Hautveränderungen sein oder zum Teil schwere Kontaktallergien. „Wobei besonders vom roten Farbstoff erhebliche Probleme ausgehen", sagt Tagungsleiter Dr. Jörg Laske, Dermatologe am Universitätsklinikum Carl Gustav Carus, Dresden. Zwar gibt es seit 2009 eine Tätowiermittelverordnung, bei der es sich überwiegend um eine Negativ-Liste von Stoffen handelt, die in den Farben nicht enthalten sein sollen. Doch beziehen viele Tätowierer keine deutschen Produkte, sondern kaufen über das Internet Farbstoffe aus

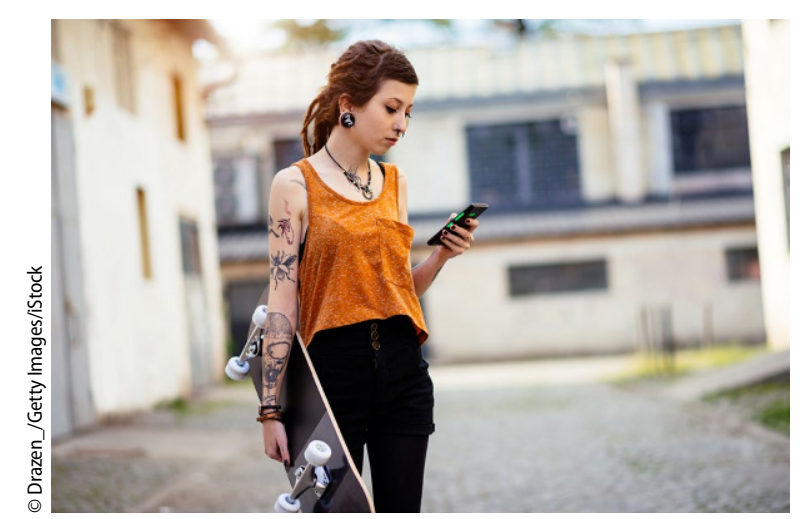

॥ Jugendliche tragen ihre Tattoos mit Stolz; was dann passiert, wenn sie entfernt werden sollen, ist Gegenstand der Forschung
Asien und den USA ein, deren Zusammensetzung nicht bekannt ist.

Auch gebe es keine Pflicht, die Rezeptur der Substanzen offenzulegen, sagt Laske. Bei schweren allergischen Reaktionen, wie sie vor allem vom roten Farbstoff ausgelöst werden können, würden schließlich oftmals weder Salben noch Laser helfen, sondern nur, das Tattoo chirurgisch zu entfernen. An unerwünschten Narben führt dann kein Weg vorbei.

\section{Akute Komplikationen und Risiken auf lange Sicht}

Mittlerweile trägt in Deutschland jeder Vierte zwischen 25 und 34 Jahren ein Tattoo. Was die Komplikationsrate betrifft, so variierten die Schätzungen zwischen zwei und $27 \%$, sagt

Dr. Roland Aschoff, Leiter der Klinik für Dermatologie am Universitätsklinikum Carl Gustav Carus. - Was akute Komplikationen, die in der Regel zwei Wochen nach der Prozedur abgeklungen sein sollten, sowie Infektionen oder auch allergische Reaktionen einschließt.

Dazu kommt aber ein anderer Aspekt: dass der Farbstoff nicht nur dort verbleibt, wo er in die Haut eingebracht wurde, sondern im Körper "wandert". Bei tätowierten Patienten mit Melanomen, schwarzem Hautkrebs, findet sich in nahgelegenen Lymphknoten der Tattoo-Farbstoff wieder, sagt Jörg Laske. „Welche Risiken das auf lange Sicht birgt, wissen wir noch gar nicht."

\section{Laserentfernung - und dann?}

Ebenfalls bisher weitgehend unbekannt sind Art und Wirkung der Abbauprodukte, die bei der Laserbehandlung entstehen, wenn nicht länger erwünschte Tätowierungen entfernt werden, sagt Roland Aschoff.
Das Prinzip der Laserentfernung: Die Pigmentartikel in der Haut zerplatzen durch Einwirken hoher Temperaturen in kleinste Einzelteile und werden anschließend über das Lymphsystem abtransportiert. „Am leichtesten zu entfernen ist der schwarze Farbstoff", erläutert Jörg Laske, „weil die dunklen Farben am meisten Energie absorbieren." Blau, Grün und - abermals - Rot erwiesen sich hier als problematisch. „Da braucht es mehr Sitzungen, und trotzdem bleiben oft Reste zurück." Welches Risiko bei der Fragmentierung von Farbpigmenten in toxische oder krebserregende Bestandteile ausgeht, ist derzeit noch Gegenstand von Forschung. Das Bundesamt für Risikobewertung hat beispielsweise für die Laserbestrahlung des Blaupigments Kupfer-Phthalocyanin flüchtige und hochgiftige Verbindungen wie Blausäure oder Benzol nachgewiesen.

\section{Technische Entwicklung}

Die technische Entwicklung geht vom bisher gebräuchlichen Nano- zum Pikolaser, der mit noch kürzeren Energie-Impulsen die Nebenwirkungen der Laserbehandlung wie Verbrennungen und Narbenbildung noch weiter mindern soll.

An den Risiken von Tattoos und deren Beseitigung ändert das allerdings nichts.

\section{Weitere Informationen:}

www.dgdc-tagung.de

Paediatr. Paedolog. Austria 2018 · 53:310 https://doi.org/10.1007/s00608018-0614-1

(c) Springer-Verlag GmbH Austria, ein Teil von Springer Nature 2018 